Bulletin of Mathematical Biology (2001) 00, 1-17

doi:10.1006/bulm.2001.0235

Available online at http://www.idealibrary.com on $\mathbf{I D E} / \mathbf{l}^{\circledR}$

\title{
Diffusion Induced Oscillatory Insulin Secretion
}

\author{
JAMES P. KEENER \\ Department of Mathematics, \\ University of Utah, \\ Salt Lake City, UT 84112, \\ U.S.A. \\ E-mail: keener@math.utah.edu
}

\begin{abstract}
Oscillatory secretion of insulin has been observed in many different experimental preparations. Here we examine a mathematical model for in vitro insulin secretion from pancreatic beta cells in a flow-through reactor. The analysis shows that oscillations result because of an important interplay between flow rate of the reactor and insulin diffusion. In particular, if the ratio of flow rate to volume of the reaction bed is too large, oscillations are eliminated, in contradiction to the conclusions of Maki and Keizer (L. W. Maki and Keizer J. Mathematical analysis of a proposed mechanism for oscillatory insulin secretion in perifused HIT-15 cells. Bull. Math. Biol., 57(1995), 569-591). Furthermore, with reasonable numbers for the experimental parameters and the diffusion of insulin, the model equations do not exhibit oscillations.
\end{abstract}

(C) 2001 Society for Mathematical Biology

\section{INTRODUCTION}

Insulin is secreted from the pancreas in an oscillatory fashion. Many in vivo and in vitro experiments have been carried out to try to understand the biochemical control mechanisms underlying these oscillations.

One particular in vitro experiment measures insulin oscillations in flow columns (Chou and Ipp, 1990; Cunningham, 1993). The apparatus for this experiment consists of a perifusion pump which pumps glucose-containing perifusate, a bed of islets or insulin secreting cells sandwiched between microcarrier beads, and a device for collecting the efflux perifusate. Because of this experimental arrangement, this system has been modeled as a one-dimensional chemical flow reactor (with no diffusion), where the reaction region is represented by the volume occupied by the cells. Under the assumption that the ratio of the length of the bed to the flow rate is small, it was suggested in Maki and Keizer (1995) that this model system could be reduced using asymptotic analysis to that of a well mixed continuously stirred tank reactor (CSTR), and therefore modeled by a system of four ordinary differential equations. Further, in this parameter range the ode system could be reduced to a system of two first-order differential equations, which could be studied in the phase plane. With these two simplifications, ranges for the glucose $0092-8240 / 01 / 000001+17 \quad \$ 35.00 / 0$ (C) 2001 Society for Mathematical Biology 
feed concentration were found for which there were stable oscillations of insulin secretion, thereby giving theoretical support for the experimental observations.

It is the claim of this paper that the reduction of a flow reactor system (without diffusion) to a CSTR is not valid and leads to the appearance of insulin oscillations that cannot exist in the (no diffusion) flow reactor. Specifically, we show that if the ratio of the length of the bed to the flow rate is small, then insulin oscillations are suppressed. On the other hand, there is a range of values of this ratio, determined by the diffusion coefficient of insulin, for which stable oscillations occur. This implies that oscillations are regulated by the interplay between the flow rate and diffusion. It also implies that the CSTR approximation is only valid when there is sufficient diffusion (or some other means of mixing).

We will develop these results in two steps. First, in the next section, we describe the standard model of a flow reactor containing insulin secreting cells. In the following section, we show that if diffusion is ignored, then for large flow rates, there are no oscillations, contrary to the claim of Maki and Keizer (1995). Finally, in the subsequent section, we modify the flow model, allowing diffusion of insulin. For this modified model, there are oscillations in certain parameter ranges, and in the limit of large diffusion the CSTR model of Maki and Keizer (1995) is recovered.

\section{THE MOdEL}

We consider the secretion of insulin from cells which are fixed in a flow reactor, with solution flowing through the reactor. The mechanism of insulin secretion that we use here is exactly the same as proposed in Maki and Keizer (1995). That is, glucose enters the cell through GLUT-type glucose transporters of two types, GLUT1 and GLUT2 transporters. Once inside the cell, internal glucose is metabolized, activating secretion via insulin granule exocytosis. In the external medium, insulin activates GLUT1 transporters and inactivates GLUT2 transporters.

To model this reaction, we assume that there is a steady flow of solution at velocity $V$ along the one-dimensional reactor, with insulin secreting cells confined to the one-dimensional region $0<x<L_{\text {bed. }}$. In the fluid we must track two concentrations, the external glucose concentration $G$ and the external insulin concentration $I$. The conservation equations for these quantities are

$$
\begin{aligned}
(1-\rho)\left(\frac{\partial G}{\partial t}+V \frac{\partial G}{\partial x}-D_{G} \frac{\partial^{2} G}{\partial x^{2}}\right) & =-R_{1}-R_{2}, \\
\frac{\partial I}{\partial t}+V \frac{\partial I}{\partial x}-D_{I} \frac{\partial^{2} I}{\partial x^{2}} & =R_{s},
\end{aligned}
$$

for $0<x<L_{\text {bed }}$, where $R_{1}$ is the rate of glucose uptake by GLUT-1 receptors, $R_{2}$ is the rate of glucose uptake by GLUT-2 receptors, and $R_{s}$ is the rate of insulin secretion by the cells in the bed, and $\rho$ is the volume fraction of cells in the bed. 
The boundary data are that $G(0, t)=G_{0}$, and $I(0, t)=0$, since the incoming flow is assumed to have fixed glucose concentration and no insulin. However, with these boundary conditions the problem is not well posed, unless diffusion is ignored. In the next section we study this problem in the case with no diffusion, in which case these boundary conditions suffice. However, in the subsequent section, where diffusion is included, these boundary conditions will be modified.

Inside the cell, glucose is metabolized at the rate $R_{m}$. Thus, if $G_{i}$ denotes the interior concentration of glucose,

$$
\rho \frac{\partial G_{i}}{\partial t}=R_{1}+R_{2}-R_{m}
$$

Notice that (3) has no derivatives with respect to $x$ since the cells are assumed to be at a fixed position in the reactor.

To complete the model description, it remains to describe the functional forms of the various rate terms. First, the rate of glucose metabolism is assumed to be an increasing function of glucose concentration. Thus,

$$
\frac{R_{m}}{\rho}=\frac{V_{m} G_{i}}{K_{m}+G_{i}},
$$

for some constants $V_{m}$ and $K_{m}$. In a similar way, the rates of the GLUT-1 and GLUT-2 transporters are assumed to be simple increasing functions of the external concentration of external glucose, $G . R_{1}$ is assumed to be an increasing function of $I$, which models the recruitment of GLUT-1 transporters by insulin, and results in positive feedback,

$$
\frac{R_{1}}{\rho}=\frac{V_{1}\left(G-G_{i}\right)}{\left(K_{1}+G_{i}\right)\left(1+G / K_{1}\right)} \frac{I}{K_{i}+I} .
$$

$R_{2}$ is assumed to be an increasing function of an inactivation variable $J$ through

$$
\frac{R_{2}}{\rho}=\frac{V_{2}\left(G J^{m}-G_{i}\right)}{\left(K_{2}+G_{i}\right)\left(1+G / K_{2}\right)} .
$$

The rate of insulin secretion is controlled by a complicated biochemical network, the details of which we do not wish to model here (Alberts et al., 1983). Instead, the insulin secretion rate $R_{s}$ is described by an empirical function determined by fitting to experimental data. By combining data on how $R_{m}$ depends on $G$ with data on how $R_{s}$ depends on $G$, one can determine the relationship between $R_{s}$ and $R_{m}$. We are then able to express $R_{s}$ in terms of $R_{m}$ and hence in terms of $G_{i}$. By doing so we circumvent the inconvenient fact that, although the rate of insulin secretion depends in some way on internal glucose concentrations, this relationship has not been measured directly. The result is

$$
R_{s}=\frac{V_{s}\left(R_{m}^{4}+\rho^{4} L^{4}\right)}{R_{m}^{4}+\rho^{4} K_{s}^{4}+\rho^{4} L^{4}} .
$$


Notice that insulin secretion is positive even when $R_{m}=0$, by virtue of the parameter $L$.

Finally we introduce a variable $J$, an inhibition variable (similar to $h$ or $n$ in the Hodgkin-Huxley context), which measures the extent to which insulin inhibits its own release. $J$ does not correspond directly to a measured physiological process, but is a phenomenological representation of the slow negative feedback process. The variable $J$ is assumed to evolve according to the differential equation

$$
\tau_{J} \frac{d J}{d t}=J_{\infty}-J
$$

where

$$
J_{\infty}=\frac{K_{\mathrm{inh}}}{K_{\mathrm{inh}}+I} .
$$

Note that $J_{\infty}$ decreases as the concentration of insulin increases, and, thus, an increase in insulin leads to a decrease in $J$, with a time delay related to the time constant $\tau_{J}$. Thus, increasing $I$ leads to a decrease in glucose flux through the GLUT2 transporters.

Most of the model parameters can be determined from experimental data, and are summarized in Table 1 . The estimated parameters, $K_{\mathrm{inh}}, K_{i}$, and $\tau_{J}$, are parameters associated with the various types of insulin feedback, which are not known. The flow rate was reported in Chou and Ipp (1990) to be $0.3 \mathrm{ml} \mathrm{\textrm {min } ^ { - 1 }}$ through a $1 \mathrm{~cm}$ diameter column, yielding a flow velocity of $3.8 \mathrm{~mm} \mathrm{~min}{ }^{-1}$. The thickness of the bed is more difficult to determine. A monolayer of islets would make $L_{\text {bed }}$ about $0.3 \mathrm{~mm}$, so that $k_{0}=\frac{V}{L_{\text {bed }}}=1.3 \mathrm{~min}^{-1}$. For a monolayer of cells, with $L_{\text {bed }}=10 \mu \mathrm{m}, k_{0}=30 \mathrm{~min}^{-1}$. In contrast, in Maki and Keizer (1995), $k_{0}$ was estimated as $400 \mathrm{~min}^{-1}$.

The first step in our analysis is to non-dimensionalize the model. We set $G=$ $K_{2} g, G_{i}=K_{2} g_{i}, I=\epsilon \frac{K_{2} V_{s}}{V_{2}} i=\frac{V_{s} L_{\text {bed }}}{V} i, x=L_{\text {bed }} y, t=\frac{K_{2}}{V_{2}} \tau, \epsilon=\frac{V_{2} L_{\text {bed }}}{V K_{2}}$, and the model becomes

$$
\begin{aligned}
\left(\frac{\partial g}{\partial \tau}+\frac{1}{\epsilon} \frac{\partial g}{\partial y}-\delta_{G} \frac{\partial^{2} g}{\partial y^{2}}\right)=( & \left.-v_{1} \frac{g-g_{i}}{\left(k_{1}+g_{i}\right)\left(k_{1}+g\right)} \frac{\epsilon i}{k_{i}+\epsilon i}-\frac{\left(g J^{m}-g_{i}\right)}{\left(1+g_{i}\right)(1+g)}\right) \\
& \times\left(\frac{\rho}{1-\rho}\right) \\
\epsilon \frac{\partial i}{\partial \tau}+\frac{\partial i}{\partial y}-\delta \frac{\partial^{2} i}{\partial y^{2}}= & \frac{r_{m}^{4}+l^{4}}{r_{m}^{4}+k_{s}^{4}+l^{4}}, \\
\frac{\partial d g_{i}}{\partial \tau}= & v_{1} \frac{g-g_{i}}{\left(k_{1}+g_{i}\right)\left(k_{1}+g\right)} \frac{\epsilon i}{k_{i}+\epsilon i}+\frac{g J^{m}-g_{i}}{\left(1+g_{i}\right)(1+g)} \\
& -v_{m} \frac{g_{i}}{k_{m}+g_{i}}, \\
\tau_{j} \frac{\partial J}{\partial \tau}= & \frac{k_{\text {inh }}}{k_{\text {inh }}+\epsilon i}-J, \quad r_{m}=\frac{g_{i}}{k_{m}+g_{i}}
\end{aligned}
$$


Table 1. Standard parameter values of the model for insulin secretion.

\begin{tabular}{|c|c|c|}
\hline \multirow[t]{11}{*}{ Fixed by experiment } & $V_{m}$ & $0.24 \mathrm{mM} \mathrm{min}^{-1}$ \\
\hline & $K_{m}$ & $9.8 \mathrm{mM}$ \\
\hline & $V_{s}$ & $0.034 \mathrm{mM} \mathrm{min}^{-1}$ \\
\hline & $K_{S}$ & $0.13 \mathrm{mM} \mathrm{min}^{-1}$ \\
\hline & $V_{1}$ & $120.0 \mathrm{mM} \mathrm{min}^{-1}$ \\
\hline & $K_{1}$ & $1.4 \mathrm{mM}$ \\
\hline & $V_{2}$ & $32.0 \mathrm{mM} \mathrm{min}^{-1}$ \\
\hline & $K_{2}$ & $17.0 \mathrm{mM}$ \\
\hline & $L$ & $0.01 \mathrm{mM} \mathrm{min}^{-1}$ \\
\hline & $D_{g}$ & $6.6 \times 10^{-6} \mathrm{~cm}^{2} \mathrm{~s}^{-1}$ \\
\hline & $D_{I}$ & $2.1 \times 10^{-6} \mathrm{~cm}^{2} \mathrm{~s}^{-1}$ \\
\hline \multirow[t]{3}{*}{ Experimentally variable } & $V$ & $3.8 \mathrm{~mm} \mathrm{~min}^{-1}$ \\
\hline & $L_{\text {bed }}$ & $0.3 \mathrm{~mm}$ \\
\hline & $G_{0}$ & $5-22 \mathrm{mM}$ \\
\hline \multirow[t]{3}{*}{ Estimated (not known) } & $K_{\text {inh }}$ & $1 \times 10^{-6} \mathrm{mM}$ \\
\hline & $K_{i}$ & $4.0 \times 10^{-5} \mathrm{mM}$ \\
\hline & $\tau_{J}$ & $20 \min$ \\
\hline
\end{tabular}

for $0<y<1$, with $g(0, \tau)=g_{0}$, and $i(0, \tau)=0$.

The analysis of this model that follows is based on the assumption that $\epsilon$ is small, in the range of $10^{-1}-10^{-4}$. Physically, this means that the flow rate is large and/or the length of the reactor bed is small. With $\epsilon$ small, we expect $\frac{\partial g}{\partial y}$ to be small, in which case $g=g_{0}$, to leading order in $\epsilon$. Also, because $\epsilon$ is small, $i$ equilibrates rapidly in $\tau$, so that $i$ can be taken to be in quasi-equilibrium. The resulting reduced system of equations is

$$
\begin{aligned}
\frac{\partial i}{\partial y}-\delta \frac{\partial^{2} i}{\partial y^{2}} & =\frac{r_{m}^{4}+l^{4}}{r_{m}^{4}+k_{s}^{4}+l^{4}}, \quad r_{m}=\frac{g_{i}}{k_{m}+g_{i}} \\
\frac{\partial g_{i}}{\partial \tau} & =v_{1} \frac{\left(g_{0}-g_{i}\right)}{\left(k_{1}+g_{0}\right)\left(k_{1}+g_{i}\right)} \frac{\epsilon i}{k_{i}+\epsilon i}+\frac{\left(g_{0} J^{m}-g_{i}\right)}{\left(1+g_{0}\right)\left(1+g_{i}\right)}-v_{m} \frac{g_{i}}{k_{m}+g_{i}} \\
& \equiv f\left(g_{i}, J, i\right) \\
\tau_{j} \frac{\partial J}{\partial \tau} & =\frac{k_{\text {inh }}}{k_{\text {inh }}+\epsilon i}-J
\end{aligned}
$$

for $0<y<1$, with $i(0, \tau)=0$. Notice that it is incorrect to set $\epsilon=0$ in (15) or (16) since $k_{i}$ and $k_{\text {inh }}$ are both significantly smaller than $\epsilon$ in the experimental parameter range. 


\section{Steady State Analysis}

In this section we examine the model equations (14)-(16) in the case of no diffusion by setting $\delta=0$ in equation (14), and show that there is a unique stable steady state solution.

To show that there is a unique steady state solution, we set derivatives with respect to $\tau$ to zero, finding

$$
\begin{aligned}
\frac{\partial i}{\partial y} & =\frac{r_{m}^{4}+l^{4}}{r_{m}^{4}+k_{s}^{4}+l^{4}} \equiv r_{s}\left(g_{i}\right), \quad r_{m}=\frac{g_{i}}{k_{m}+g_{i}} \\
0 & =v_{1} \frac{\left(g_{0}-g_{i}\right)}{\left(k_{1}+g_{0}\right)\left(k_{1}+g_{i}\right)} \frac{\epsilon i}{k_{i}+\epsilon i}+\frac{\left(g_{0} J^{m}-g_{i}\right)}{\left(1+g_{0}\right)\left(1+g_{i}\right)}-v_{m} \frac{g_{i}}{k_{m}+g_{i}} \\
J & =\frac{k_{\text {inh }}}{k_{\text {inh }}+\epsilon i}=J_{\infty}(i) .
\end{aligned}
$$

It is clear from (15) that $g_{i}$ cannot exceed $g_{0}$, since $\frac{\partial g_{i}}{\partial \tau}<0$ if $g_{i}=g_{0}$. Observe that (18) can be solved to find $J^{m}$ as a function of $g_{i}$ and $i$,

$$
\begin{aligned}
g_{0} J^{m} & =g_{i}+\frac{\left(1+g_{0}\right)\left(1+g_{i}\right)}{v_{2}}\left(v_{m} \frac{g_{i}}{k_{m}+g_{i}}-\frac{\left(g_{0}-g_{i}\right)}{\left(k_{1}+g_{0}\right)\left(k_{1}+g_{i}\right)} \frac{\epsilon i}{k_{i}+\epsilon i}\right) \\
& \equiv H\left(g_{i}, i\right) .
\end{aligned}
$$

The function $H\left(g_{i}, i\right)$ is a monotonically increasing function of $g_{i}$ for each fixed $i$, and $H(0, i)<0$ while $H\left(g_{0}, i\right)>g_{0}$. Thus, the equation

$$
g_{0} J_{\infty}^{m}(i)=H\left(g_{i} ; i\right)
$$

has a unique solution $g_{i}=h(i)$, for each positive $i$. It follows from (17) that there is a unique solution profile $i(y)$ with $i(0)=0$ which is monotonically increasing in $y$ and satisfies the differential equation

$$
\frac{\partial i}{\partial y} \equiv r_{s}(h(i)), \quad i(0)=0 .
$$

Sketches of the steady profiles of insulin and internal glucose are shown in Figs 1 and 2. It is apparent from these that the uptake of glucose in different regions of the reactor is by different mechanisms. At the front end of the reactor (near $x=0$ ) where there is little insulin, the GLUT-2 receptors are most active. As insulin increases down the reactor, however, these receptors are inhibited and GLUT-1 receptors are activated, so that at the far end of the reactor (near $x=1$ ) the uptake of insulin is entirely through GLUT-1 receptors. 


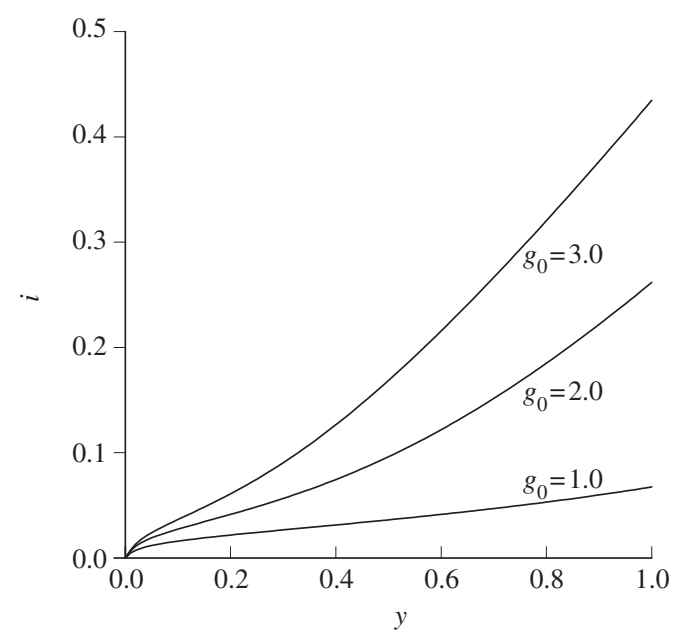

Figure 1. The steady distribution of insulin $i(y)$ for three different values of glucose $g_{0}=1.0,2.0,3.0$.

3.1. Linear stability. The goal of this section is to establish conditions under which the unique steady state solution is linearly stable. First, it is easy to see that the steady solution is stable if $\epsilon$ is sufficiently small. In the limit of small $\epsilon$, the dynamics become trivial,

$$
\begin{aligned}
\frac{\partial i}{\partial y} & =\frac{r_{m}^{4}+l^{4}}{r_{m}^{4}+k_{s}^{4}+l^{4}}, \quad r_{m}=\frac{g_{i}}{k_{m}+g_{i}}, \\
\frac{\partial g_{i}}{\partial \tau} & =\frac{\left(g_{0} J^{m}-g_{i}\right)}{\left(1+g_{0}\right)\left(1+g_{i}\right)}-v_{m} \frac{g_{i}}{k_{m}+g_{i}}, \\
\tau_{J} \frac{\partial J}{\partial \tau} & =1-J,
\end{aligned}
$$

so that $J \rightarrow 1$, and $g_{i} \rightarrow$ constant, independent of $i$, a boring (non-oscillatory) solution.

Physically, it makes intuitive sense that the system should be stable if $\epsilon$, which is inversely proportional to the flow rate, is small, since then all insulin that is produced is rapidly removed from the reactor. In this parameter regime, the GLUT2 transporters never inactivate and the GLUT1 transporters never activate.

For a more general stability analysis, we linearize the system (14)-(16) about the steady state, finding the linear system

$$
\begin{aligned}
& \frac{\partial u}{\partial y}=\alpha v, \\
& \frac{\partial v}{\partial \tau}=-\beta v+\gamma u+\delta w,
\end{aligned}
$$




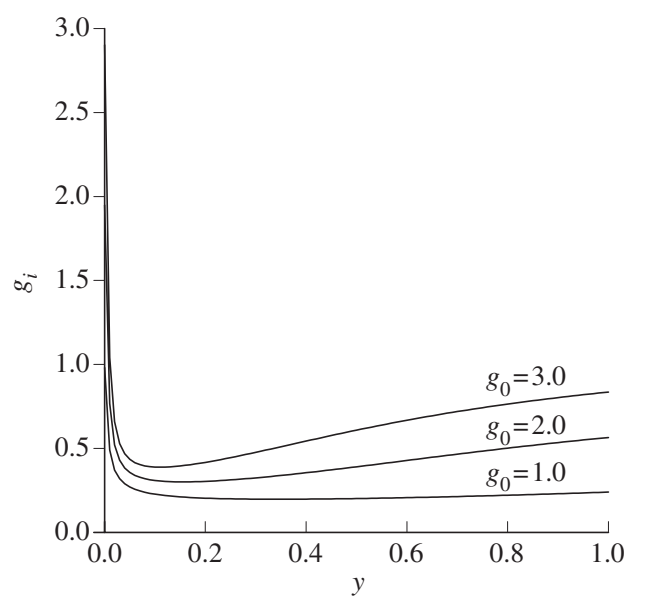

Figure 2. The steady distribution of internal glucose $g_{i}(y)$ for three different values of external glucose $g_{0}=1.0,2.0,3.0$.

$$
\tau_{j} \frac{\partial w}{\partial \tau}=-\eta u-w
$$

where

$$
\begin{aligned}
& \alpha=\frac{\partial r_{s}}{\partial g_{i}} \\
& \beta=-\frac{\partial f}{\partial g_{i}}=v_{1} \frac{1}{\left(k_{1}+g_{i}\right)^{2}} \frac{\epsilon i}{k_{i}+\epsilon i}+\frac{1+g_{0} J^{m}}{\left(1+g_{0}\right)\left(1+g_{i}\right)^{2}}+v_{m} \frac{k_{m}}{\left(k_{m}+g_{i}\right)^{2}} \\
& \gamma=\frac{\partial f}{\partial i}=v_{1} \frac{\left(g_{0}-g_{i}\right)}{\left(k_{1}+g_{0}\right)\left(k_{1}+g_{i}\right)} \frac{\epsilon k_{i}}{\left(k_{i}+\epsilon i\right)^{2}} \\
& \delta=\frac{\partial f}{\partial J}=\frac{m g_{0} J^{m-1}}{\left(1+g_{0}\right)\left(1+g_{i}\right)}, \\
& \eta=-\frac{\partial J_{\infty}(i)}{\partial i}=\frac{\epsilon k_{\text {inh }}}{\left(k_{\text {inh }}+\epsilon i\right)^{2}}
\end{aligned}
$$

evaluated at the steady solution $i(y)$. Thus, each of these parameters are functions of $y$. Notice also that each of these parameters is positive.

We simplify this linear system somewhat by introducing the variables

$$
V=\alpha v, \quad W=\frac{w}{\eta}, \quad \sigma=\frac{\tau}{\tau_{j}}
$$

yielding the linear system

$$
\frac{\partial u}{\partial y}=V
$$




$$
\begin{aligned}
& \frac{\partial V}{\partial \sigma}=-a V+b u+c W, \\
& \frac{\partial W}{\partial \sigma}=-u-W,
\end{aligned}
$$

subject to the boundary condition $u(0, \sigma)=0$, where $a=\tau_{j} \beta, b=\tau_{j} \alpha \gamma$, $c=\tau_{j} \alpha \delta \eta$.

Now we construct a Lyapunov function for this system. Notice that

$$
\frac{\partial}{\partial \sigma}\left((a+1) V^{2}+2 c V W+b c W^{2}\right)=-A V^{2}-B W^{2}+C u V,
$$

where $A=2(a+1) a, B=2 c(b-c)$, and $C=2(a+1) b-2 c$. Observe that

$$
\begin{aligned}
\int_{0}^{1} C u V d y & =\int_{0}^{1} C u u_{y} d y \\
& =\frac{1}{2} C(1) u^{2}(1)-\frac{1}{2} \int_{0}^{1} C_{y} u^{2} d y \\
& =\frac{1}{2} C(1)\left(\int_{0}^{1} V d y\right)^{2}-\frac{1}{2} \int_{0}^{1} C_{y}\left(\int_{0}^{y} V d \xi\right)^{2} d y
\end{aligned}
$$

and invoking the Schwarz inequality, we find

$$
\begin{aligned}
\int_{0}^{1} C u V d y & \leq \frac{1}{2} C(1) \int_{0}^{1} V^{2} d y+\frac{1}{2} \int_{0}^{1}\left|C_{y}\right| d y \int_{0}^{1} V^{2} d y \\
& =\frac{1}{2} \int_{0}^{1} D V^{2} d y
\end{aligned}
$$

where

$$
D=C(1)+\int_{0}^{1}\left|C_{y}\right| d y .
$$

Integrating (38), we find that

$$
\begin{aligned}
\frac{d}{d \sigma} \int_{0}^{1}\left((a+1) V^{2}+2 c V W+b c W^{2}\right) d y & =-\int_{0}^{1}\left(A V^{2}+B W^{2}\right) d y+\int_{0}^{1} C u V d y \\
& \leq-\int_{0}^{1}\left(\left(A-\frac{1}{2} D\right) V^{2}+B W^{2}\right) d y
\end{aligned}
$$

We now have a proof of stability if:

(i) the Lyapunov function

$$
L=\int_{0}^{1}\left((a+1) V^{2}+2 c V W+b c W^{2}\right) d y
$$

is positive definite, 
(ii) $A>\frac{1}{2} D$ for all $y$, and

(iii) $B>0$ for all $y$,

since then, $\frac{d L}{d \sigma} \leq 0$ whenever $L>0$, which implies that $L \rightarrow 0$ as $\sigma \rightarrow \infty$.

The Lyapunov function is positive definite whenever

$$
b(a+1)>c,
$$

and $B>0$ whenever

$$
b>c .
$$

Clearly, (43) is satisfied whenever (44) is satisfied.

Notice that $a, b$ and $c$ are all proportional to $\tau_{j}$, so that (43) is satisfied for large enough $\tau_{j}$. Notice also that both $\gamma$ and $\eta$ are proportional to $\epsilon$ and so vanish in the limit that $\epsilon \rightarrow 0$. It follows that $D$ is small whenever $\epsilon$ is small, so that $a>\frac{1}{2} D$ for all sufficiently small $\epsilon$. Thus, we have stability for all small $\epsilon$, provided $b>c$, or equivalently, provided $\gamma>\delta \eta$.

In Fig. 3 is shown a sketch of $\gamma-\delta \eta$ for $g_{0}=1.0$ and $\epsilon=4.9 \times 10^{-3}$, and all other parameters as in Table 2. Unfortunately, for these parameter values, $\gamma-\delta \eta$ is not strictly positive, and so the proof of linear stability given here fails. There are several possible ways to salvage something. The first is to change the model equations slightly so that the proof of stability works. This is not particularly difficult to do, since the failure of the proof to apply is due to the sensitivity of $J_{\infty}$ to $i$ for small values of $i$. Since the function $J_{\infty}$ is not known, changing it slightly has little effect on the faithfulness of the model to known reality. Here, the function $J_{\infty}$ was used solely because it is the same as was used in Maki and Keizer (1995). The second way to assess the stability of the steady solution is with extensive numerical simulations. Indeed, numerical simulations verify that the steady solution is stable, so we take this as satisfactory evidence for stability.

\section{Diffusion}

It appears that to obtain oscillations, one must include diffusion. Thus, we consider the model system

$$
\begin{aligned}
\frac{\partial i}{\partial y}-\delta \frac{\partial^{2} i}{\partial y^{2}}= & \frac{r_{m}^{4}+l^{4}}{r_{m}^{4}+k_{s}^{4}+l^{4}} \equiv r_{s}\left(g_{i}\right), \quad r_{m}=\frac{g_{i}}{k_{m}+g_{i}}, \\
\frac{\partial g_{i}}{\partial \tau}= & v_{1} \frac{\left(g_{0}-g_{i}\right)}{\left(k_{1}+g_{0}\right)\left(k_{1}+g_{i}\right)} \frac{\epsilon i}{k_{i}+\epsilon i}+\frac{\left(g_{0} J^{m}-g_{i}\right)}{\left(1+g_{0}\right)\left(1+g_{i}\right)} \\
& -v_{m} \frac{g_{i}}{k_{m}+g_{i}}, \\
\tau_{j} \frac{\partial J}{\partial \tau}= & \frac{k_{\text {inh }}}{k_{\text {inh }}+\epsilon i}-J,
\end{aligned}
$$




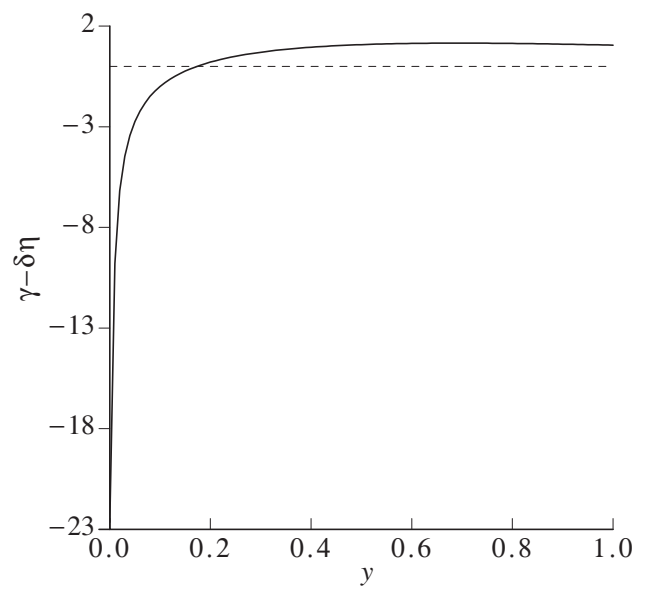

Figure 3. $\gamma-\delta \eta$ as a function of $y$ for $g_{0}=1.0$ and $\epsilon=4.9 \times 10^{-3}$.

for $0<y<1$. Our first challenge is to determine appropriate boundary conditions for $i$.

Outside the interval $0<y<1, i$ must satisfy $\frac{\partial i}{\partial y}-\delta \frac{\partial^{2} i}{\partial y^{2}}=0$. Solutions of this equation are of the form $i=A+B e^{y / \delta}$. The only solution with $i$ bounded as $y \rightarrow \infty$ is $i=A$, so we must have $\frac{\partial i}{\partial y}(1)=0$. For $y<0$, however, all solutions are bounded, so we simply require $i(0)=\delta \frac{\partial i}{\partial y}(0)$.

Now consider the solution of the equation

$$
\frac{\partial i}{\partial y}-\delta \frac{\partial^{2} i}{\partial y^{2}}=r_{s}
$$

subject to these boundary conditions, in the limit that $\delta$ is large. We let $\alpha=1 / \delta$ and write

$$
\frac{\partial^{2} i}{\partial y^{2}}=\alpha\left(\frac{\partial i}{\partial y}-r_{s}\right)
$$

subject to

$$
\frac{\partial i}{\partial y}(0)=\alpha i(0), \quad \frac{\partial i}{\partial y}(1)=0
$$

Now a power series expansion in $\alpha, i=i_{0}+\alpha i_{1}+O\left(\alpha^{2}\right)$ yields the hierarchy of equations

$$
\begin{aligned}
& \frac{\partial^{2} i_{0}}{\partial y^{2}}=0, \quad \frac{\partial i_{0}}{\partial y}(0)=0, \quad i_{0} \frac{\partial i}{\partial y}(1)=0 \\
& \frac{\partial^{2} i_{1}}{\partial y^{2}}=\frac{\partial i_{0}}{\partial y}-r_{s}, \quad \frac{\partial i_{1}}{\partial y}(0)=i_{0}(0), \quad \frac{\partial i_{1}}{\partial y}(1)=0 .
\end{aligned}
$$


Table 2. Standard nondimensional parameter values for the model of insulin secretion.

\begin{tabular}{lccl}
\hline Fixed by experiment & $v_{m}$ & $\frac{V_{m}}{V_{2}}$ & 0.0075 \\
& $k_{m}$ & $\frac{K_{m}}{K_{2}}$ & 0.58 \\
& $l$ & $\frac{L}{V_{m}}$ & 0.04 \\
& $k_{S}$ & $\frac{K_{s}}{V_{m}}$ & 0.54 \\
& $k_{1}$ & $\frac{K_{1}}{K_{2}}$ & 0.08 \\
Experimentally variable & $v_{1}$ & $\frac{K_{1} V_{1}}{K_{2} V_{2}}$ & 0.31 \\
& $\epsilon$ & $\frac{V_{2} L_{\text {bed }}}{V K_{2}}$ & \\
& $g_{0}$ & $\frac{G_{0}}{K_{2}}$ & $0.5-1.5$ \\
& $\delta_{G}$ & $\frac{D_{G} K_{2}}{V_{2} L_{\text {bed }}^{2}}$ & \\
& $\delta$ & $\frac{D_{I}}{V L_{\text {bed }}}$ & \\
Estimated & $k_{\text {inh }}$ & $K_{\text {inh }} \frac{V_{2}}{K_{2} V_{s}}$ & $5.5 \times 10^{-5}$ \\
& $k_{i}$ & $\frac{K_{i} V_{2}}{V_{s} K_{2}}$ & $2.2 \times 10^{-3}$ \\
& $\tau_{j}$ & $\tau_{J} \frac{V_{2}}{K_{2}}$ & 37.6 \\
\hline & & &
\end{tabular}

The solution of the first of these is clearly $i_{0}=$ constant. Then, from the second we learn that

$$
\frac{\partial i_{1}}{\partial y}=-\int_{0}^{y} r_{s} d y+i_{0},
$$

and the requirement that $\frac{\partial i_{1}}{\partial y}(1)=0$ implies that

$$
i_{0}=\int_{0}^{1} r_{s} d y
$$

Notice, however, that if $i$ is well approximated by a constant, then $g_{i}$ is nearly a constant also, in which case $r_{s}$ is constant, $i_{0}=r_{s}\left(g_{i}\right)$, and the system reduces to

$$
\begin{aligned}
\frac{d g_{i}}{d \tau} & =v_{1} \frac{\left(g_{0}-g_{i}\right)}{\left(k_{1}+g_{0}\right)\left(k_{1}+g_{i}\right)} \frac{\epsilon i}{k_{i}+\epsilon i}+\frac{\left(g_{0} J^{m}-g_{i}\right)}{\left(1+g_{0}\right)\left(1+g_{i}\right)}-v_{m} \frac{g_{i}}{k_{m}+g_{i}}, \\
\tau_{j} \frac{d J}{d \tau} & =\frac{k_{\text {inh }}}{k_{\text {inh }}+\epsilon i}-J,
\end{aligned}
$$

where

$$
i=\frac{g_{i}}{k_{m}+g_{i}},
$$

which is the system studied by Maki and Keizer (1995). 


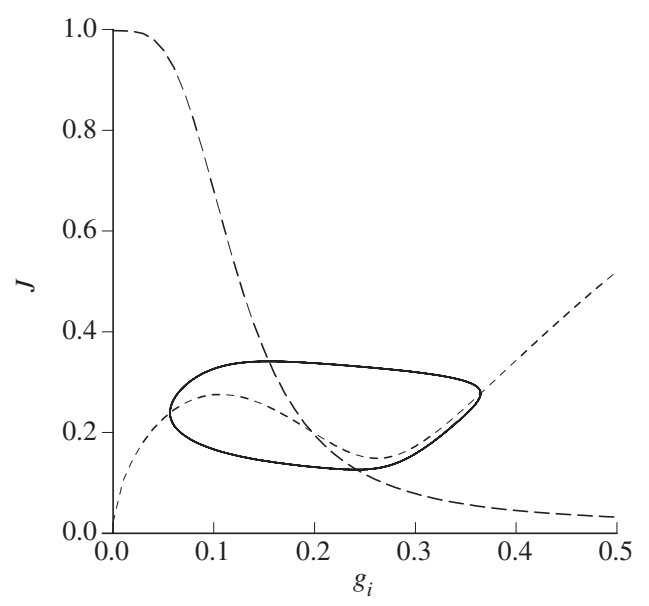

Figure 4. Phase portrait for the two variable system (55)-(56), shown with $g_{0}=0.9$. The nullclines $\frac{d J}{d t}=0$ and $\frac{d g_{i}}{d t}=0$ are shown as long and short dashed curves, respectively.

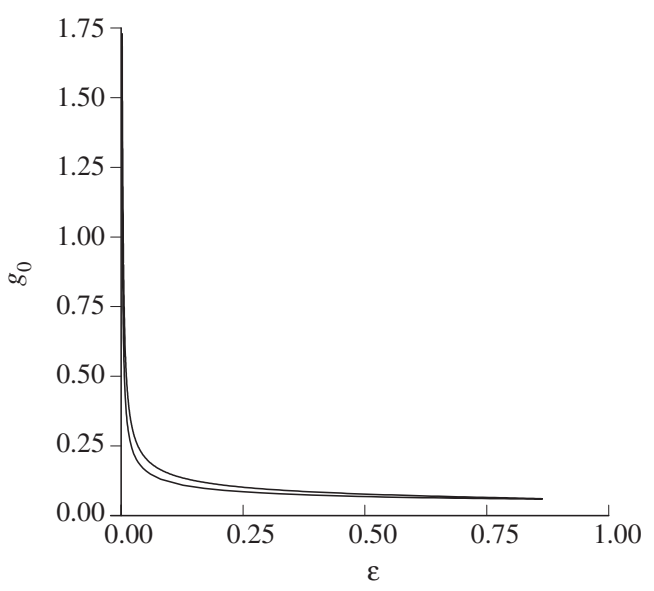

Figure 5. Curve of Hopf bifurcation points for the ode system (55)-(56) in the $g_{0}-\epsilon$ parameter plane.

The observation of Maki and Keizer is that this system has periodic solutions which arise from Hopf bifurcations. The reason for these Hopf bifurcations is clear, revealed by a phase portrait.

In Fig. 4 is shown a phase portrait for this system with $g_{0}=0.9$. The nullclines $\frac{d J}{d t}=0$ and $\frac{d g_{i}}{d t}=0$ are shown as long and short dashed curves respectively, and the periodic solution is shown as the solid curve.

Because of the ' $\mathrm{N}$-shaped' nature of the $\frac{d g_{i}}{d t}=0$ nullcline, we expect there to be two values of $g_{0}$ at which there are Hopf bifurcations. That this is the case is verified by Fig. 5, wherein is shown the curve of Hopf bifurcation points plotted in the $g_{0}$ versus $\epsilon$ parameter plane. The significant feature of this plot is that for a finite range of positive $\epsilon$, there are two Hopf bifurcation points. For values of $g_{0}$ between these, the two variable system has a stable periodic solution. 


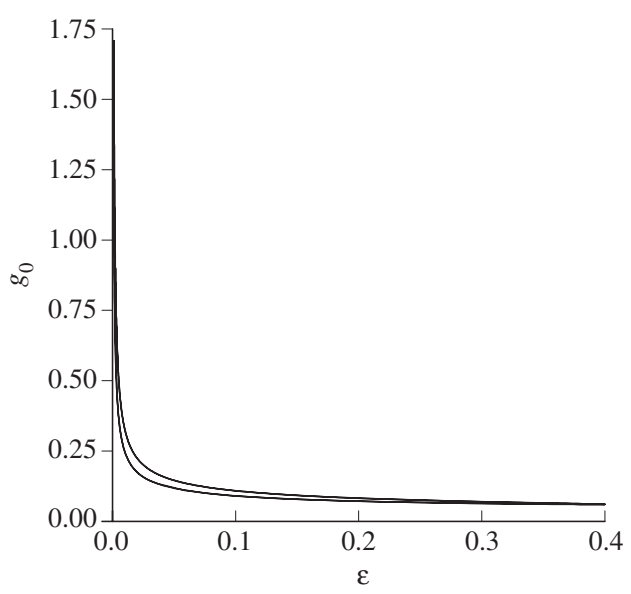

Figure 6. Curve of Hopf bifurcation points for the pde system with $\delta=1.0$ in the $g_{0}-\epsilon$ parameter plane.

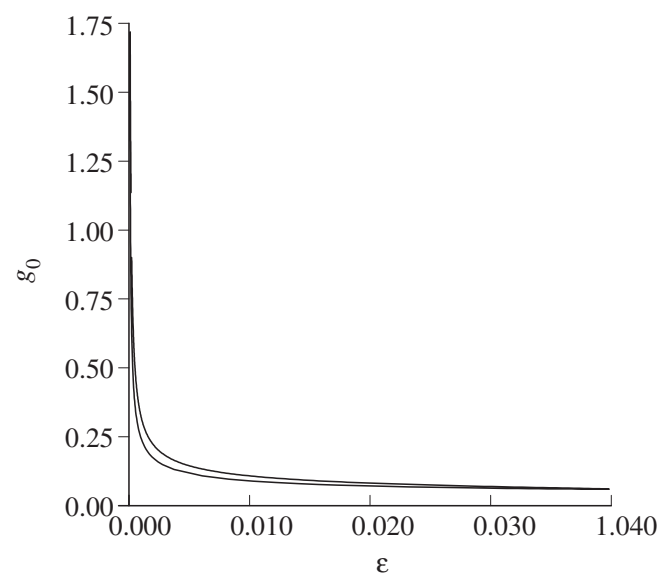

Figure 7. Curve of Hopf bifurcation points for the pde system with $\delta=0.25$ in the $g_{0}-\epsilon$ parameter plane.

This two variable system was found as the limiting system in the limit of large $\delta$. Therefore, we expect this same behavior to occur for the pde system with $\delta$ finite, even though a phase portrait for the pde system cannot be drawn. That this is the case is shown in Figs 6 and 7, where the curves in the $g_{0}-\epsilon$ parameter plane at which there is a Hopf bifurcation are shown. In Fig. $6, \delta=1.0$ whereas in Fig. 7, $\delta=0.25$. Notice that the size of the region in which there are periodic solution decreases as $\delta$ becomes smaller. (All of the computations in this section were done using AUTO97 (Doedel et al., 1997).)

A second way to examine the Hopf bifurcations of this system is in the $g_{0^{-}}$ $\delta$ parameter plane, holding $\epsilon$ fixed. Slicing the Hopf bifurcation surface in this way, there are two characteristic behaviors. For $\epsilon$ sufficiently large, curves are as depicted in Fig. 8, while for smaller values of $\epsilon$ they are as in Fig. 9. In Fig. 10 is 


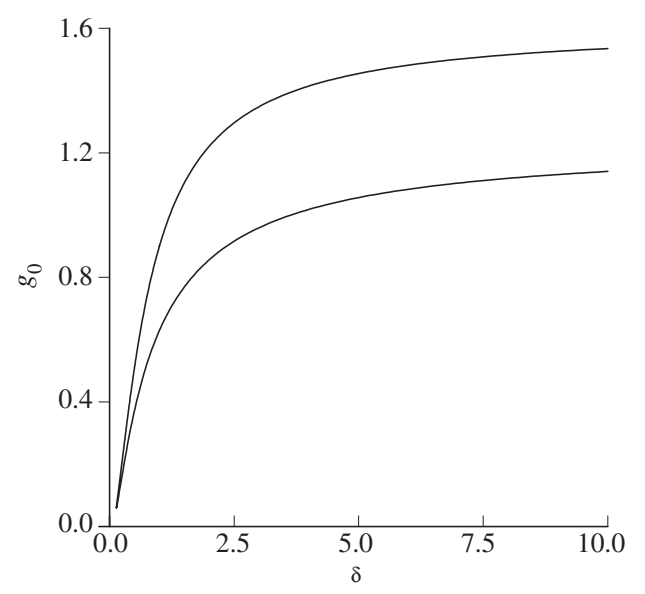

Figure 8. Hopf bifurcation curve in the $g_{0^{-}} \delta$ parameter plane with $\epsilon=2.3 \times 10^{-3}$.

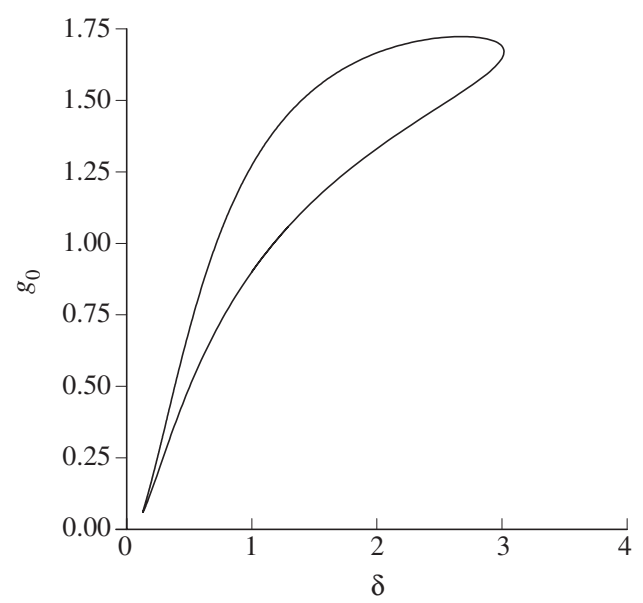

Figure 9. Hopf bifurcation curve in the $g_{0}-\delta$ parameter plane with $\epsilon=1.5 \times 10^{-3}$.

shown the boundary of Hopf bifurcation points in the $\delta-\epsilon$ plane. Above this curve the system has stable oscillatory solutions, whereas below this curve the steady solution is stable and there are no oscillatory solutions. This region actually has two boundaries but because of the scale the leftmost boundary cannot be seen in Fig. 10. For this reason, a blowup of the same region is shown in Fig. 11. From this plot it is apparent that the steady solution is stable and there are no oscillatory solutions if $\delta$ is sufficiently small, less than about 0.1 .

\section{Conclusion}

In this problem there are two parameters that determine the behavior of the system. In the limit $\epsilon \rightarrow 0$ (the flow dominated limit) the dynamics are trivial, 


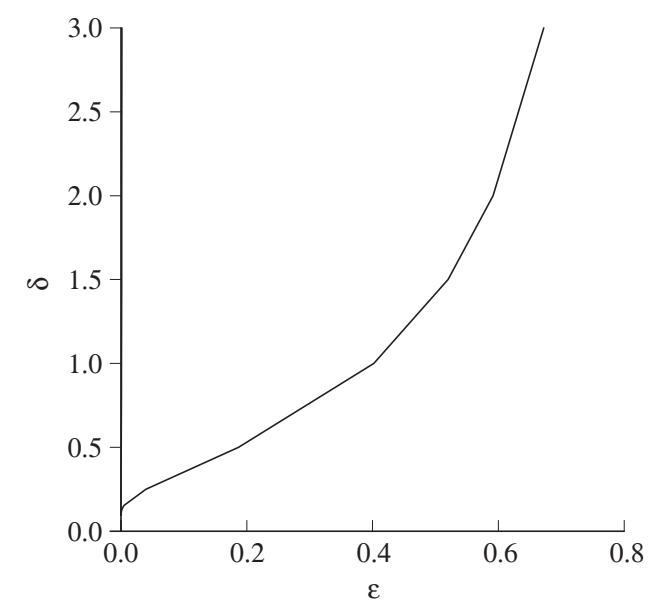

Figure 10. The boundary of Hopf bifurcation points in the $\delta-\epsilon$ parameter plane.

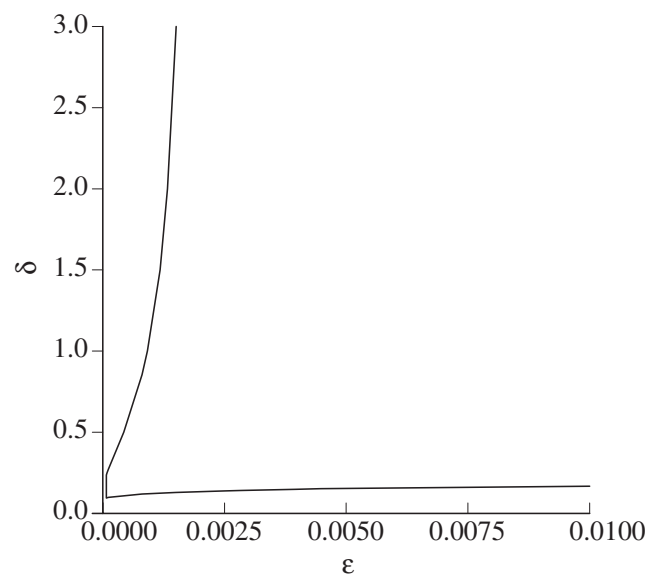

Figure 11. Blowup of the boundary of Hopf bifurcation points in the $\delta-\epsilon$ parameter plane near the $\epsilon=0$ axis.

approaching a stable steady state. Furthermore, with no diffusion, there are no oscillations for any value of $\epsilon$. In the limit $\delta \rightarrow \infty$ (the diffusion dominated limit), there is oscillatory behavior for $g_{0}$ in the right range. Furthermore, there are oscillations for ranges of $g_{0}$ with large enough diffusion, provided $\epsilon$ is not too small. Thus, oscillations occur when there is sufficient diffusion to adequately mix the concentrations in the reacting layer of cells. If there is insufficient mixing (as occurs with very high flow rates), then the oscillation is inhibited. That oscillations are inhibited at high flow rates is to be expected for the simple reason that upstream cells cannot know what downstream cells are doing, and so upstream cells cannot be inhibited by high levels of insulin that are only experienced by downstream cells.

Having observed that sufficient diffusion is required for insulin oscillations, we are faced with an unresolved dilemma. Using reasonable estimates for the exper- 
imental parameters $L_{\text {bed }}$ and $V$, and a reasonable diffusion coefficient for insulin (see Table 1), we estimate that $\delta=1.8 \times 10^{-4}$, a number that is far too small to induce diffusion driven oscillations in this model. (Numerical solutions suggest a lower bound for $\delta$ on the order of 0.1 .) In other words, adding physically realistic diffusion to the model does not account for the observed oscillations. However, a further dilemma is that without diffusion it is difficult to understand how the cellular oscillations can be synchronized across the reactor bed, which was 0.5 $\mathrm{cm}$ in radius. Perhaps the fluid dynamics of the reactor bed was much more complicated than is assumed in this simple model, and diffusion was substantially enhanced by fluid mixing, but the observation stands that insulin oscillations cannot occur in a flow reactor unless there is sufficient diffusion (mixing) to overcome the unidirectional flow.

\section{ACKNOWLEDGEMENT}

This research is supported in part by NSF grant DMS-99700876.

\section{REFERENCES}

Alberts, B., D. Bray, J. Lewis, M. Raff, K. Roberts and J. D. Watson (1983). Molecular Biology of the Cell, New York: Garland.

Chou, H. F. and E. Ipp (1990). Pulsatile insulin secretion in isolated rat islets. Diabetes 39, 112-117.

Cunningham, B. A. (1993). Characterization of oscillations in insulin secretion, $\mathrm{PhD}$ thesis, Boston University.

Doedel, E. J., A. R. Champneys, T. F. Fairgrieve, Y. A. Kuznetsov, B. Sandstede and X. J. Wang (1997). AUTO97: Continuation and Bifurcation Software forOrdinary Differential Equations (with HomCont), User's Guide. ftp.cs.concordia/pub/ doedel/auto.

Maki, L. W. and J. Keizer (1995). Mathematical analysis of a proposed mechanism for oscillatory insulin secretion in perifused HIT-15 cells. Bull. Math. Biol. 57, 569-591. 